\title{
EXTREMAL GRAPHS OF ORDER DIMENSION 4
}

\author{
GEIR AGNARSSON
}

\begin{abstract}
We study the maximal number of edges of a graph on $p$ vertices of order dimension 4 . We will show that the lower bound for this number is greater than $\frac{3}{8} p^{2}+2 p-13$. In particular the Turán4 graph on $p$ vertices does not have the maximal number of edges among the graphs of order dimension 4.
\end{abstract}

\section{Introduction}

Although the main result in this article is stated graph theoretically, it was discovered in an algebraic and geometric setting. We start by discussing the graph theoretical view point, then the geometric one, and finally the original algebraic one, together with a brief history.

Graph theory. Any order of a given set $X$ can be viewed as a subset $P$ of $X \times X$, which satisfies certain conditions. By a partially ordered set, or simply a poset, we mean a tuple $(X, P)$ where $P$ defines a partial order of $X$. The order dimension of this poset is defined as the least number $n$ of linear orders (or total orders) $L_{1}, \ldots, L_{n}$ of $X$ such that $P=L_{1} \cap \cdots \cap L_{n}$ as a subset of $X \times X$. We say that the linear orders $L_{1}, \ldots, L_{n}$ realize the poset $(X, P)$. For every simple finite graph $G=(V, E)$, we can view $V \cup E$ as a poset, where the partial order is defined by letting each pair of distinct vertices and each pair of distinct edges be incomparable, and by letting each edge be greater than its endvertices and no other vertices. The order dimension of our graph $G=(V, E)$ is then the order dimension of the poset $V \cup E$ as described above. A more comprehensive treatment can be found in William T. Trotter's treatise [8].

Geometry. Perhaps this view will explain the use of the word dimension here better. Assume that a graph $G$ has order dimension $n$, and is realized by linear orders $L_{1}, \ldots, L_{n}$. Let $\mathrm{N}_{0}=\{0,1,2, \ldots\}$ and provide the cartesian product $\mathrm{N}_{0}^{n}$ with the usual component-wise partial order. If we list the elements

Received January 18, 1999. 
of $V$ increasingly w. r. t. $L_{i}$ for each $i$, then, for each $v \in V$, let $l_{i}(v)$ be the place number of $v$ in the list $L_{i}$. Consider the map $\rho: V \rightarrow \mathrm{N}_{0}^{n}$ defined by

$$
\rho(v)=\left(l_{1}(v), \ldots, l_{n}(v)\right) .
$$

This map is an embedding of $V$ into $\mathrm{N}_{0}^{n}$, such that

1. $\rho(V)$ is a set of incomparable elements of $\mathrm{N}_{0}^{n}$.

2. For each edge $e=\{u, v\}$ in $G$, the corresponding join satisfies $\rho(u) \vee$ $\rho(v) \geq \rho(w) \Leftrightarrow w \in\{u, v\}$.

In this way we get a natural geometrical realization of $G$ as a sub-poset in $\mathrm{N}_{0}^{n}$. Conversely, every collection of incomparable points in $\mathrm{N}_{0}^{n}$ corresponds to some graph $G$ as a poset, by letting the points correspond to vertices and by letting each join of two points, that is not greater than any other point than those two, correspond to an edge.

Since the example provided in the proof of our main result, Theorem 2.1, will be presented in geometrical form, let us explain what we will do, before discussing the algebraic point of view.

For natural numbers, $n$ and $p$, let $e_{n}(p)$ be the maximal number of edges of a graph of order dimension $n$, on $p$ vertices. The purpose of this paper is to give a lower bound for $e_{4}(p)$. First let us consider the cases $n \leq 3$. By the definition of order dimension, we see that $e_{1}(p)=1$, and that a connected graph has order dimension $\leq 2$ if and only if it is a simple path. Therefore we have $e_{2}(p)=p-1$. A strong theorem by Schnyder, [8, Theorem (2.1) p. 128], states that a graph has order dimension $\leq 3$ if and only if it is planar. This theorem, together with Eulers formula [5, Corollary 4.2.8], which relates the number of vertices, edges and regions formed by a planar embedding of the graph, yields that $e_{3}(p)=3 p-6$. Hence, for $n \leq 3$, we know the values of $e_{n}(p)$ for all $p \geq 1$. The first case where no exact formula is known, is $n=4$. Clearly $e_{4}(p)$ is bounded from above by $\left(\begin{array}{l}p \\ 2\end{array}\right)$, the number of edges in the complete graph, $K_{p}$, on $p$ vertices. We will, in the last section, discuss the upper bound better. In the second section we provide a class of concrete examples of points in $\mathrm{N}_{0}^{n}$ such that for $p \geq 8$ we have

$$
e_{4}(p) \geq \frac{p^{2}+5 p-24}{2}-\left(\left\lfloor\frac{p}{4}\right\rfloor+1\right)\left(p-2\left\lfloor\frac{p}{4}\right\rfloor\right)
$$

where $\lfloor x\rfloor$ denotes the largest integer $\leq x$. This implies that $e_{4}(p)>\frac{3}{8} p^{2}+$ $2 p-13$ for all $p \geq 8$.

Algebra. Because it is easier, and more natural from an algebraic point of view, we will consider a geometrical object called filter generated by a 
collection of points in $\mathrm{N}_{0}^{n}$, and then count the number of outer corners of this filter:

Definition 1.1. Let $n$ be a natural number.

1. A filter in $\mathrm{N}_{0}^{n}$ is a subset $U$ of $\mathrm{N}_{0}^{n}$ such that $U+\mathrm{N}_{0}^{n} \subseteq U$.

2. The outer corners of a filter $U$ in $\mathrm{N}_{0}^{n}$ are the maximal points (w.r.t. the component-wise partial order) that are not contained in $U$.

If $k$ is a field we note that the semigroup consisting of monomials of the $k$-algebra $R=k\left[X_{1}, \ldots, X_{n}\right]$ is isomorphic to the additive semigroup $\mathrm{N}_{0}^{n}$, via the isomorphism $\phi\left(X_{1}^{i_{1}} \cdots X_{n}^{i_{n}}\right)=\left(i_{1}, \ldots, i_{n}\right)$. With this isomorphism, we get a one-to-one correspondence between monomial ideals of $R$ and filters of $\mathrm{N}_{0}^{n}$.

Before discussing the algebraic aspect, let us display some definitions that we will use in our discussion. For a given monomial ideal $M$, we say that a generator is minimal if it is contained in every set of generators for $M$.

Definition 1.2. Let $k$ be a field, and let $M$ be a monomial ideal of the $k$-algebra $R=k\left[X_{1}, \ldots, X_{n}\right]$.

1. The ideal $M$ is artinian if each indeterminate $X_{i}$ appears to some power in $M$.

2. The ideal $M$ is generic if no variable $X_{i}$ appears with the same non-zero exponent in two distinct minimal generators of $M$.

3. The ideal $M$ is generic artinian if it is both generic and artinian.

Note. $M$ is artinian if and only if the quotient algebra $R / M$ has finite dimension as a vector space over $k$.

We now explain briefly how our problem of determining the maximal number of edges of a graph of order dimension $n$ on $p$ vertices, is the same as finding the maximal number of 1-faces of a simplicial complex of order dimension $n$ on $p$ vertices, and is therefore just a small part of a much more fundamental algebraic question.

As we saw earlier, every graph $G=(V, E)$ of order dimension $n$, has an embedding, as a poset, in $\mathrm{N}_{0}^{n}$ via $\rho$. The points in the set $\rho(V)$ are incomparable and generate a filter in $\mathrm{N}_{0}^{n}$, and hence $\left(\phi^{-1} \circ \rho\right)(V)$ is a set of minimal generators for a monomial ideal $M_{G}$ of $k\left[X_{1}, \ldots, X_{n}\right]$, which, as described in detail in [3], yields a very special kind of simplicial complex called Scarf complex, due to Herbert Scarf, who first defined it in [6]. A Scarf complex of a monomial ideal $M \subseteq k\left[X_{1}, \ldots, X_{n}\right]$ with minimal generators $W_{1}, \ldots, W_{p}$, is an abstract simplicial complex $\Delta_{M}$ on $\{1, \ldots, p\}$ of dimension $\leq n-1$, constructed in the following way: For a subset $S \subseteq\{1, \ldots, p\}$ let $W_{S}$ be the least common multiple of $W_{i}, i \in S$. We let $S$ be a face of $\Delta_{M}$ if 
1. for all $i \notin S, W_{i}$ does not divide $W_{S}$, and

2. for all $i \in S$, the support of $W_{S} / W_{i}$ is a strict subset of $\left\{X_{1}, \ldots, X_{n}\right\}$.

In a Scarf complex derived from a graph, the vertices of the graph correspond to the vertices (or 0-faces) of the complex, and the edges of the graph correspond to the 1-faces of the complex. According to [3, sections 5 and 6], the maximal number $\beta_{k}(n, p)$ of $k$-faces of a simplicial complex of order dimension $n$ on $p$ vertices, is obtained among the Scarf complexes of monomial ideals which are generated by $p$ monomials in $n$ variables, and which can be assumed to be generic artinian. Therefore, to determine the maximal number of edges of a graph on $p$ vertices of order dimension $n$ is the same as finding the maximal number of 1-faces of a simplicial complex on $p$ vertices of order dimension $n$. Hence, this problem is a special case of a fundamental algebraic question posed by Prof. Bernd Sturmfels [3] of U. C. Berkeley: Determine how many $k$-faces a simplicial complex on $p$ vertices of order dimension $n$ can have.

Recall the correspondence $\phi$ above. For a monomial ideal $M$ of $R$, let $S$ be a face of $\Delta_{M}$ and $B_{S}$ be the $n$-dimensional hyper-box in $\mathrm{N}_{0}^{n}$ spanned by $\phi\left(W_{S}\right)$. From the definition of $\Delta_{M}$ we see that $S$ is a face of $\Delta_{M}$ if $B_{S}$ is the least hyper-box containing $\left\{\phi\left(W_{i}\right): i \in S\right\}$ in its boundary (i.e. the union of the $(n-1)$-dimensional sides of $\left.B_{S}\right)$, and $B_{S}$ contains no other $\phi\left(W_{j}\right)$. It turns out that for each maximal simplex $S^{*}$ of the Scarf complex $\Delta_{M}$, the points $\phi\left(W_{i}\right)$, $i \in S^{*}$, bound exactly one outer corner point of the filter $\phi(M)$. Hence, the facets, or the maximal simplices of $\Delta_{M}$, are in one-to-one correspondence with the outer corners of the filter $\phi(M)$. In the proof of Theorem 2.1, it is exactly the outer corners of the resulting filter that we count.

If $c_{n}(p)$ is the maximal number of outer corners of a filter in $\mathrm{N}_{0}^{n}$ generated by $p$ or fewer points, then we soon will see that $c_{4}(p)$ and $e_{4}(p)$ both grow quadratically in $p$, and their difference is a linear polynomial in $p$.

We can view the present paper as a continuation of [1], where it is shown that $c_{4}(p)$ is not a quadratic polynomial for $p \geq 4$. One of the main conclusions [1, Theorem 35] is that for $p \geq 4$ we have

$$
\begin{array}{ll}
c_{4}(p) & =\frac{p^{2}-3 p-2}{2} \quad \text { for } p \in\{4,5, \ldots, 12\}, \\
c_{4}(13) & =63, \\
c_{4}(p) \leq \frac{p^{2}-3 p-4}{2} & \text { for } p \geq 13 .
\end{array}
$$

We note that $c_{4}(p)$ is given by a quadratic polynomial for $4 \leq p \leq 12$. We then get a "break-point" at $p=13$, where previous polynomial expression does not hold. 
This led the algebraist Bernd Sturmfels to establish a relation with William T. Trotter's graph theory result, that the order dimension of the complete graph on 12 vertices, $K_{12}$, is 4 , but the order dimension of $K_{13}$ is 5 [7]. We now attempt to explain how: Given an integer $p \geq 4$. We bear in mind the correspondence between filters in $\mathrm{N}_{0}^{4}$ and monomial ideals in four variables. That the order dimension of $K_{p}$ is 4 , is, according to our previous discussion, equivalent to the existence of a generic artinian monomial ideal $M$, generated by $p$ elements in 4 variables, whose Scarf-complex $\Delta_{M}$ has precisely $p$ vertices and $\left(\begin{array}{c}p \\ 2\end{array}\right)$ edges [3, Theorem 6.5, p. 13]. The corresponding simplicial Scarf-polytope $P_{M}$ whose boundary is $\Delta_{M}$, has therefore also $f_{0}\left(P_{M}\right)=p$ vertices and $f_{1}\left(P_{M}\right)=\left(\begin{array}{c}p \\ 2\end{array}\right)$ edges. If $f_{k}\left(P_{M}\right)$ is the number of $k$-faces of the Scarf polytope $P_{M}$ in the case $n=4$, then we get by the Dehn-Sommerville equations [9, p. 252] that

$$
\begin{aligned}
f_{0}\left(P_{M}\right)-f_{1}\left(P_{M}\right)+f_{2}\left(P_{M}\right)-f_{3}\left(P_{M}\right) & =0, \\
f_{2}\left(P_{M}\right)-2 f_{3}\left(P_{M}\right) & =0 .
\end{aligned}
$$

From these equations we get that the number of facets of $P_{M}$ is $f_{3}\left(P_{M}\right)=$ $f_{1}\left(P_{M}\right)-p=\left(p^{2}-3 p\right) / 2$, and hence the outer corner points of $M$ are one less, or $f_{3}\left(P_{M}\right)-1=\left(p^{2}-3 p-2\right) / 2$. Since now the number of outer corner points of $M$ is $\leq c_{4}(p)$ we have by (1.2) that $p \leq 12$.

By carrying on in the setup from above, we have in fact for arbitrary $p \geq$ 4, that a generic artinian monomial ideal $M$ in 4 variables generated by $p$ monomials with $c_{4}(p)$ outer corner points, has a Scarf polytope $P_{M}$ with $f_{3}\left(P_{M}\right)=c_{4}(p)+1$ facets, and hence by the Dehn-Sommerville equations it has $f_{1}\left(P_{M}\right)=f_{3}\left(P_{M}\right)+p=\left(c_{4}(p)+1\right)+p$ edges. We have therefore the following:

Proposition 1.3. The maximal number, $e_{4}(p)$, of edges of a graph on $p$ vertices which has order dimension 4 is $c_{4}(p)+p+1$.

\section{The lower bound}

Recall that if $x$ is a real number then $\lfloor x\rfloor$ denotes the largest integer $\leq x$. We have the following:

THEOREM 2.1. For $p \geq 8$ we have

$$
c_{4}(p) \geq \frac{p^{2}+3 p-26}{2}-\left(\left\lfloor\frac{p}{4}\right\rfloor+1\right)\left(p-2\left\lfloor\frac{p}{4}\right\rfloor\right) .
$$

Proof. Note that the statement of this theorem is equivalent to say that for 
each $q \geq 2$ we have

$$
\begin{aligned}
c_{4}(4 q) & \geq 6 q^{2}+4 q-13, \\
c_{4}(4 q+1) & \geq 6 q^{2}+7 q-12, \\
c_{4}(4 q+2) & \geq 6 q^{2}+10 q-10 \quad \text { and } \\
c_{4}(4 q+3) & \geq 6 q^{2}+13 q-7 .
\end{aligned}
$$

Hence it suffices to provide examples of filters generated by $4 q, 4 q+1,4 q+2$ and $4 q+3$ points in $\mathrm{N}_{0}^{4}$ that have $6 q^{2}+4 q-13,6 q^{2}+7 q-12,6 q^{2}+10 q-10$ and $6 q^{2}+13 q-7$ outer corner points, respectively.

A filter $U_{4 q}$ generated by $4 q$ points:

$(4 q-3,0,0,0),(3 q-2-i, 3 q-3+i, 2 q-1-i, i) i=1, \ldots, q-1$, $(0,4 q-3,0,0),(3 q-3+i, 3 q-2-i, i, 2 q-1-i)-$,

$(0,0,4 q-3,0),(i, 2 q-1-i, 3 q-3+i, 3 q-2-i)-$,

$(0,0,0,4 q-3),(2 q-1-i, i, 3 q-2-i, 3 q-3+i)-$.

A filter $U_{4 q+1}$ generated by $4 q+1$ points:

$(4 q-2,0,0,0),(3 q-1-i, 3 q-3+i, 2 q-i, i) \quad i=1, \ldots, q$,

$(0,4 q-2,0,0),(3 q-2+i, 3 q-2-i, i, 2 q-i) \quad i=1, \ldots, q-1$,

$(0,0,4 q-2,0),(i, 2 q-1-i, 3 q-2+i, 3 q-1-i)-$,

$(0,0,0,4 q-2),(2 q-1-i, i, 3 q-1-i, 3 q-2+i)-$.

A filter $U_{4 q+2}$ generated by $4 q+2$ points:

$(4 q-1,0,0,0),(3 q-1-i, 3 q-2+i, 2 q+1-i, i) i=1, \ldots, q$,

$(0,4 q-1,0,0),(3 q-2+i, 3 q-1-i, i, 2 q+1-i)-$,

$(0,0,4 q-1,0),(i, 2 q-1-i, 3 q-1+i, 3 q-i) \quad i=1, \ldots, q-1$,

$(0,0,0,4 q-1),(2 q-1-i, i, 3 q-i, 3 q-1+i) \quad-$

A filter $U_{4 q+3}$ generated by $4 q+3$ points:

$(4 q, 0,0,0),(3 q-i, 3 q-1+i, 2 q+1-i, i) i=1, \ldots, q$,

$(0,4 q, 0,0),(3 q-1+i, 3 q-i, i, 2 q+1-i)-$,

$(0,0,4 q, 0),(i, 2 q-i, 3 q-1+i, 3 q+1-i)-$,

$(0,0,0,4 q),(2 q-i, i, 3 q-i, 3 q+i) \quad i=1, \ldots, q-1$.

These filters, $U_{4 q}, U_{4 q+1}, U_{4 q+2}$ and $U_{4 q+3}$, turn out to have $6 q^{2}+4 q-$ $13,6 q^{2}+7 q-12,6 q^{2}+10 q-10$ and $6 q^{2}+13 q-7$ outer corner points respectively.

For a filter $U$ in $\mathrm{N}_{0}^{4}$, let $M_{U}$ be the unique corresponding monomial ideal of the $k$-algebra $R=k\left[X_{1}, X_{2}, X_{3}, X_{4}\right]$, and let $\mathbf{m}$ be the maximal ideal 
generated by the four indeterminates of $R$. Recall that $\left(\mathbf{m}: M_{U}\right)$ is the ideal of $R$ consisting of all the elements $r \in R$ with $\mathbf{m} r \in M_{U}$. By the definition of $\mathbf{m}$, we see that $\left(\mathbf{m}: M_{U}\right)$ consists of all $r \in R$, satisfying $X_{i} r \in M_{U}$ for each $i \in\{1,2,3,4\}$. Hence, $\left(\mathbf{m}: M_{U}\right)$ is a monomial ideal of $R$, whose basis, as a vector space over $k$, consists of the monomials of $M_{U}$, together with those monomials $r=X_{1}^{a_{1}} X_{2}^{a_{2}} X_{3}^{a_{3}} X_{4}^{a_{4}} \in R$, which are such, that when we increase any power $a_{i}$ by at least 1 , then the resulting element will fall in $M_{U}$. It is precisely these elements that correspond to the outer corners of $U$. Therefore, the corresponding ideal $\left(\mathbf{m}: M_{U}\right) / M_{U}$ in the quotient $k$-algebra $R / M_{U}$, has a $k$-basis which is in one to one correspondence with the outer corners of the filter $U$. We conclude that the number of outer corner points of $U$ is $\operatorname{dim}_{k}\left(\left(\mathbf{m}: M_{U}\right) / M_{U}\right)$, which can be calculated using a computer algebra system. The package [4] will be more than sufficient.

By Proposition 1.3 and Theorem 2.1 we get the following corollary:

COROLlary 2.2. For $p \geq 8$ we have that

$$
e_{4}(p) \geq \frac{p^{2}+5 p-24}{2}-\left(\left\lfloor\frac{p}{4}\right\rfloor+1\right)\left(p-2\left\lfloor\frac{p}{4}\right\rfloor\right) .
$$

The above corollary implies in particular that $e_{4}(p)>\frac{3}{8} p^{2}+2 p-13$ for all $p \geq 8$.

\section{Final remarks}

We now discuss what consequences the result in previous section has in graph theoretic setting.

Definition 3.1. Let $n$ and $p$ be positive integers, and assume $p=q n+r$ where $r \in\{0,1, \ldots, n-1\}$. The Turán-n graph, $T^{n}(p)$, is a complete $n$-partite graph on $p$ vertices that has $n-r$ parts of size $q$, and $r$ parts of size $q+1$.

Consider now the Turán-4 graph, $T^{4}(p)$, on $p$ vertices for $p \geq 8$. By [2, Theorem 3.1], every 4-partite graph has order dimension at most 4 , and hence $T^{4}(p)$, being a 4-partite graph, has order dimension 4. If $t_{4}(p)$ denotes the the number of edges in $T^{4}(p)$, and $p=4 q+r$, where $r \in\{0,1,2,3\}$, then

$$
t_{4}(p)=\left(\begin{array}{c}
4-r \\
2
\end{array}\right) q^{2}+\left(\begin{array}{l}
r \\
2
\end{array}\right)(q+1)^{2}+r(4-r) q(q+1) .
$$

Turán's theorem states in particular that the maximal number of edges of a graph that does not contain $K_{5}$, the complete graph of size 5 as subgraph, is $t_{4}(p)$. By considering all the values of $r$, where $p=4 q+r$, we see that 
$e_{4}(p)-t_{4}(p) \geq 2 p-12$ for all $p \geq 8$ and hence the set of Turán-4 graphs on $p$ vertices is strictly contained in the set of graphs of order dimension 4 on $p$ vertices.

Remark 3.2. (K. Reuter): Since the order dimension of $K_{13}$ is 5, every graph of order dimension 4 can not contain $K_{13}$ as a subgraph, and is therefore a subgraph of the Turán-12 graph. The maximal number, $t_{12}(p)$, of edges of the Turán-12 graph on $p$ vertices satisfies $\lim _{p \rightarrow \infty} t_{12}(p) / p^{2}=11 / 24$ [5, Lemma 7.1.4]. Hence $\lim \sup e_{4}(p) / p^{2} \leq 11 / 24$. So for large $p$, the set of graphs of order dimension 4 , on $p$ vertices, is strictly contained in the set of all Turán-12 graphs on $p$ vertices.

A stronger asymptotic result than in the above remark, can be found in [2], where it is shown that $\lim _{p \rightarrow \infty} e_{4}(p) / p^{2}=3 / 8$. This supports the statement that equality might actually hold in the inequality stated in Corollary 2.2.

ACKNOWLEDGEMENTS. The author would like to thank Klaus Reuter and Bernd Sturmfels for their valuable help improving the introduction of this paper. Thanks also to Günter M. Ziegler for his encouragement.

\section{REFERENCES}

1. Agnarsson, Geir, The Number of Outside Corners of Monomial Ideals, J. Pure Appl. Algebra 117 \& 118 (1997), 3-21.

2. Agnarsson, G., Felsner, S., Trotter, W. T., The Maximum Number of Edges in a Graph of Bounded Dimension, with Applications To Ring Theory, Discrete Math. 201 (1999), Issue no. 1-3, p. 5-19.

3. Bayer, Dave, Peeva, Irena and Sturmfels, Bernd, Monomial Resolutions, Math. Res. Lett. 5 (1998), p. 31-46, MR 99c:13029.

4. Bayer, Dave, Stillman, Michael, Macaulay: a computer algebra system available by anonymous ftp from zariski.harvard.edu. (1987).

5. Diestel, Reinhard, Graph Theory, Grad. Texts in Math. 173 (1997).

6. Scarf, Herbert, The Computation of Economic Equilibria, Cowles Foundation Monograph 24, Yale University Press, (1973).

7. Trotter, William T., Some Combinatorial Problems for Permutations, Congr. Numer. 19 (1978), 619-632.

8. Trotter, William T., Combinatorics and Partially Ordered Sets, Dimension Theory, Johns Hopkins Ser. Math. Sci. (1992).

9. Ziegler, Günter M., Lectures on Polytopes, Grad. Texts in Math. 152 (1995).

SCIENCE INSTITUTE

UNIVERSITY OF ICELAND

REYKJAVÍK

ICELAND

E-mail: geira@ raunvis.hi.is 